\title{
Close Range Spectral Imaging for Disease Detection in Plants Using Autonomous Platforms: a Review on Recent Studies
}

\author{
Puneet Mishra $^{1}$ - Gerrit Polder ${ }^{1}$ - Nastassia Vilfan ${ }^{1}$
}

Published online: 2 April 2020

(C) The Author(s) 2020

\begin{abstract}
Purpose of Review A short introduction to the spectral imaging (SI) of plants along with a comprehensive overview of the recent research works related to disease detection in plants using autonomous phenotyping platforms is provided. Key benefits and challenges of SI for plant disease detection on robotic platforms are highlighted.

Recent Findings SI is becoming a potential tool for autonomous platforms for non-destructive plant assessment. This is because it can provide information on the plant pigments such as chlorophylls, anthocyanins and carotenoids and supports quantification of biochemical parameters such as sugars, proteins, different nutrients, water and fat content. A plant suffering from diseases will exhibit different physicochemical parameters compared with a healthy plant, allowing the SI to capture those differences as a function of reflected or absorbed light.

Summary Potential of SI to non-destructively capture physicochemical parameters in plants makes it a key technique to support disease detection on autonomous platforms. SI can be broadly used for crop disease detection by quantification of physicochemical changes in the plants.
\end{abstract}

Keywords Imaging spectroscopy $\cdot$ Plant phenotyping $\cdot$ Plant pathogen detection $\cdot$ NIRS $\cdot$ Non-destructive $\cdot$ Spectroscopy Rapid $\cdot$ Automation $\cdot$ Phenotyping

\section{Introduction}

Worldwide, plant diseases lead to major yield and quality losses in agriculture production systems [1]. Diseases are mainly caused by pathogenic microorganisms such as fungi, viruses, bacteria, mycoplasmas and protozoa [2]. Typically, diseases start with the introduction of a vector or infested plant material at a location, which in time spreads to neighbouring plants leading to significant damage, even before it is visible to humans. For detection of pathogens, different molecular

This article belongs to the Topical Collection on Agriculture Robotics.

Puneet Mishra

puneet.mishra@wur.nl

Gerrit Polder

gerrit.polder@wur.nl

Nastassia Vilfan

nastassia.rajhvilfan@wur.nl

1 Agro-Food Robotics, Wageningen University and Research, P.O. Box 17, 6700 AA Wageningen, The Netherlands and serological methods, such as enzyme-linked immunosorbent assay (ELISA) and polymerase chain reaction (PCR), can be used to target specific proteins and deoxyribose nucleic acid (DNA) sequences, respectively.

These molecular and serological methods provide a highly specific detection of pathogens; however, they are destructive and limited to the laboratory and require highly skilled manpower. Despite such detection being of high interest to scientific research towards understanding the plant-pathogen interactions, these methods cannot be used in situ for real-time detection of diseases in plants before the symptoms are visible. Furthermore, these destructive methods cannot be used to follow the progress of the disease or the effect of crop protection compounds that are being used to control the disease spread. Early detection of disease or disease symptoms using nondestructive sensors is of high interest as it can directly benefit the agricultural systems by reducing associated losses [3-5].

Early non-destructive disease detection in plants is a key emerging topic [6]. To accomplish this task, autonomous platforms are increasingly being implemented [7, 8]. An autonomous agricultural platform in field conditions can be understood as a robotic vehicle with a range of sensors integrated to 
it (an example: http://www.agrointelli.com/). An autonomous platform in greenhouse conditions can be understood as an automated platform supporting plant growth and monitoring with a range of sensors (an example: https://www.psb.ugent. be/phenotyping/phenovision). With advancements in nondestructive sensing and computing power, autonomous platforms are providing results and conclusions in real time, which were previously limited to days of experiments. In recent years, promising applications of spectral imaging (SI) are emerging for detecting disease in plants in the open field as $[9 \bullet, 10 \bullet]$ combination of two complementary sensing modalities, i.e. spectroscopy and imaging, where the imaging captures the physical shape and structure of the plants and the spectroscopy captures complementary chemical properties of plants. In all, SI provides distribution of physicochemical properties of plants brought by the disease in a spatially preserving way.

In the present review, a small introduction of SI and what it can detect in relation to plant disease is explained. Furthermore, a comprehensive overview of the recent research related to disease detection in plants by using autonomous robotic platforms is provided. Key benefits and challenges of SI for plant disease detection, either in the greenhouse or in the open field, are highlighted, and future directions are outlined.

\section{What Is Spectral Imaging?}

SI combines traditional imaging with spectroscopy. Unlike colour red, green and blue (RGB) imaging SI refers to a group of techniques which captures more than just the red, green and blue wavelength bands. SI can be explored in different parts of electromagnetic radiation (EMR) ranging from the highenergy X-rays to the very low-energy microwaves [11]. A typical SI setup is composed of three main components: a light source, a spectrograph or filter system, and a detector for image generation. Based on the spectral range to be explored, the components are selected to meet the selectivity and sensitivity. The images generated by SI are three dimensional (3D) with two spatial $(x \times y)$ and one spectral dimension (z). Particularly, in the spectral range of 400-2500 nm, the data is highly collinear and requires advanced multi-variate statistical tools to extract and model patterns. A better understanding of SI and data analysis can be found in recent reviews [12••, 13].

\section{What Does Spectral Imaging Detect in Diseased Plants?}

Plants, unlike animals, lack an adaptive immune system limiting their ability to adapt to diseases based on the memories from past infections [14]. However, due to a highly evolved genome, plants possess a wide range of resistance activities which can counteract the disease-causing pathogens. The resistance activities are activated as soon as the plant receives a signal about coming in contact with a potential vector. Plant defence includes morphological and structural changes at the cell wall, with a massive and directed reorganisation within the host cells; changes in gene expression and general metabolism $[15,16]$.

At first, the symptoms can be identified locally, where the vector interacted with the host tissue: early physiological and structural changes reflect a deviation in photosynthetic activity. Local symptoms might include changes in concentrations of photosynthetic pigments such as chlorophylls, changes in water content and an increase in plant defence-response metabolites. Changes in cell structure at the site of infection can also be noted utilising microscopic techniques. SI in the spectral range of $400-2500 \mathrm{~nm}$ is of high interest, as it can provide access to plant biochemical parameters. Specifically, the visible (VIS) part (400$700 \mathrm{~nm}$ ) provides information on the plant pigments, such as chlorophylls, anthocyanins and carotenoids, and the near-infrared (NIR) (700-2500 nm) supports quantification of biochemical parameters, such as sugars, proteins, water and fat contents, and cell structure of plant tissues $[12 \bullet, 17,18]$. SI captures this information by measuring the percent of light being reflected/absorbed at specific wavelengths. For example, chlorophyll absorption peaks at 465 and $665 \mathrm{~nm}$, while water absorbs at approximately 1400 and $1900 \mathrm{~nm}$. With a change in plant physiological status, the presence of these specific compounds will change, influencing the absorption in relevant spectral bands. Diseased tissue exhibits clear differences in physicochemical properties compared with a healthy tissue, which allows the SI to capture those differences in the function of reflected or absorbed light (400-2500 nm). Therefore, the information captured by SI can be extracted to develop models for disease detection and severity quantification.

\section{Recent Applications}

A large number of applications can be found that utilise SI for disease detection (Lowe et al., 2017) [19]. However, there exist a limited number of applications, where SI has been integrated with automated agricultural platforms. A summary of recent applications of SI for disease detection (past 5 years) is provided in Table 1. A separate column is presented to highlight if the study utilised an autonomous platform. A study excluding an autonomous platform can be understood as a future direction for the implementation of the diseasedetection technique in autonomous platforms. 


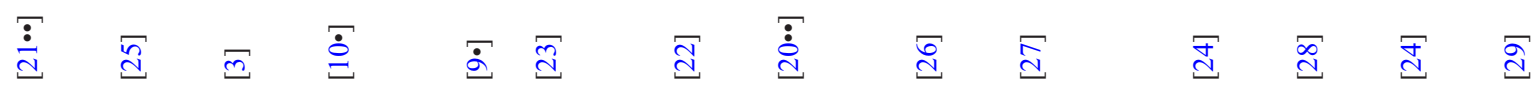

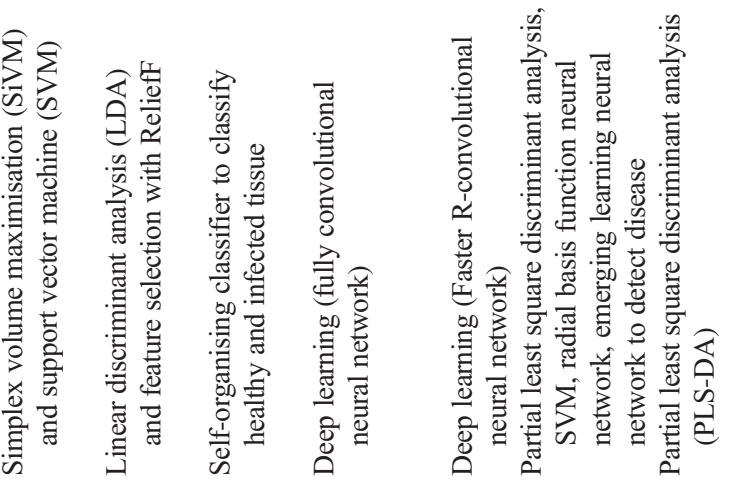

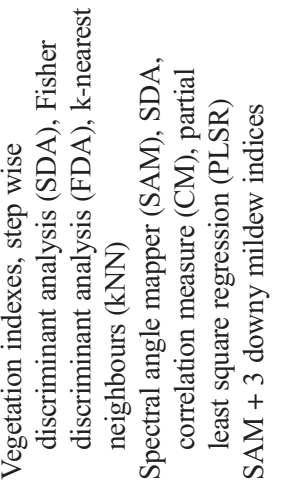

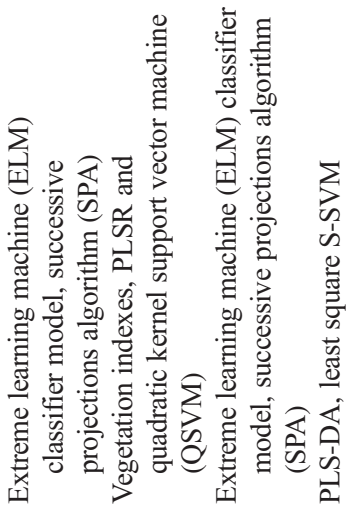

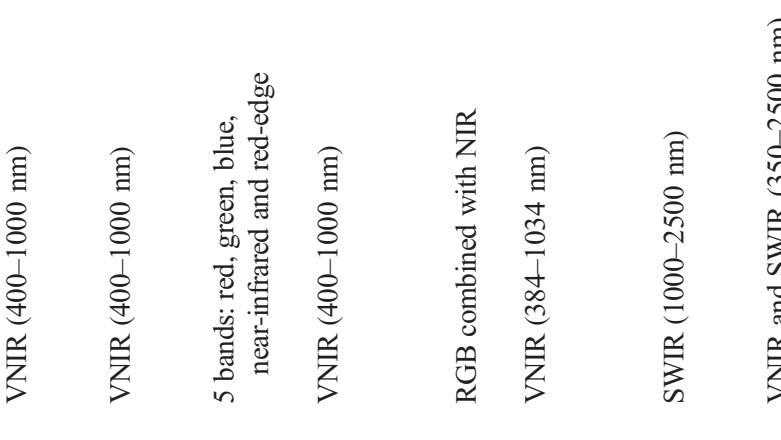

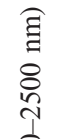

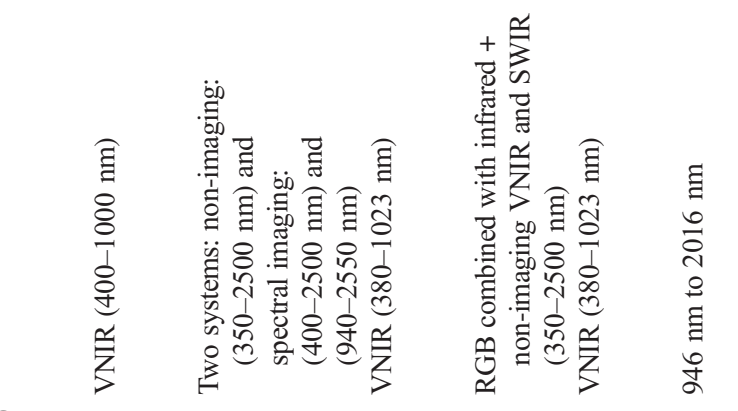

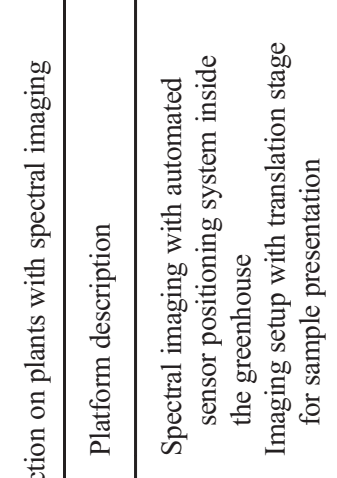

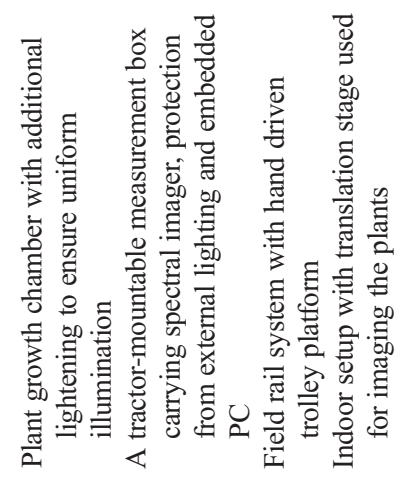

,

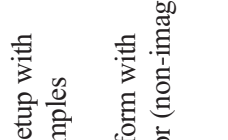

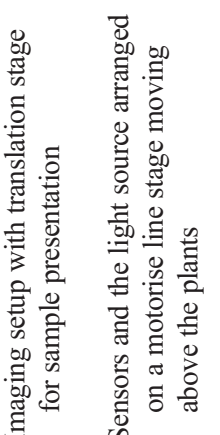

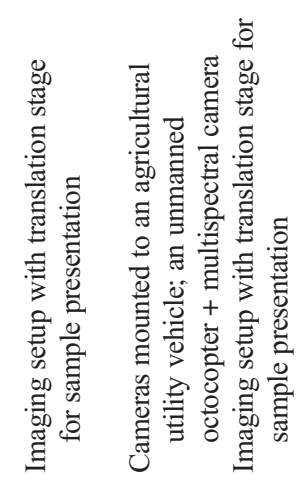

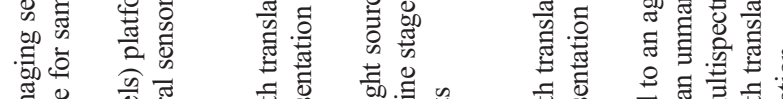

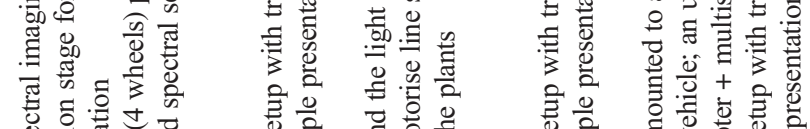

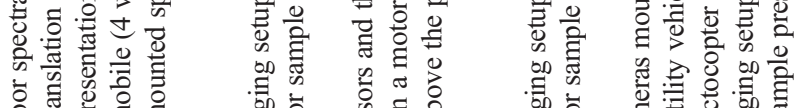

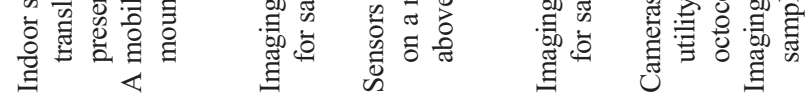

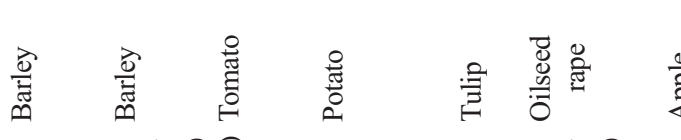

竞

章

章 1

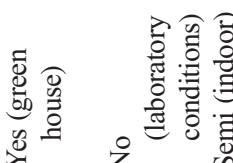

翠

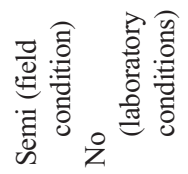

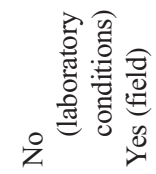

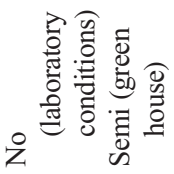

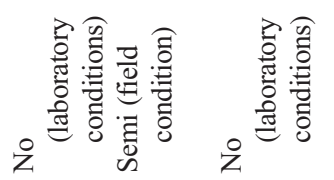

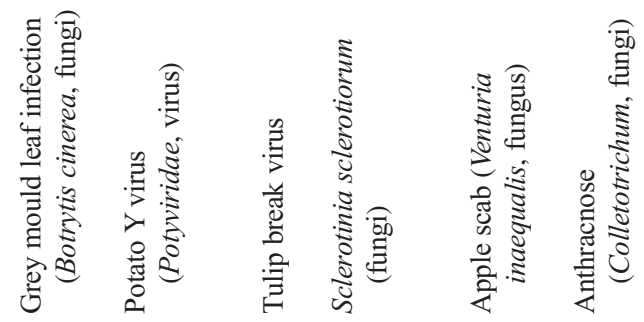

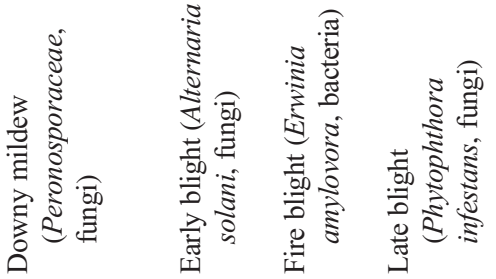




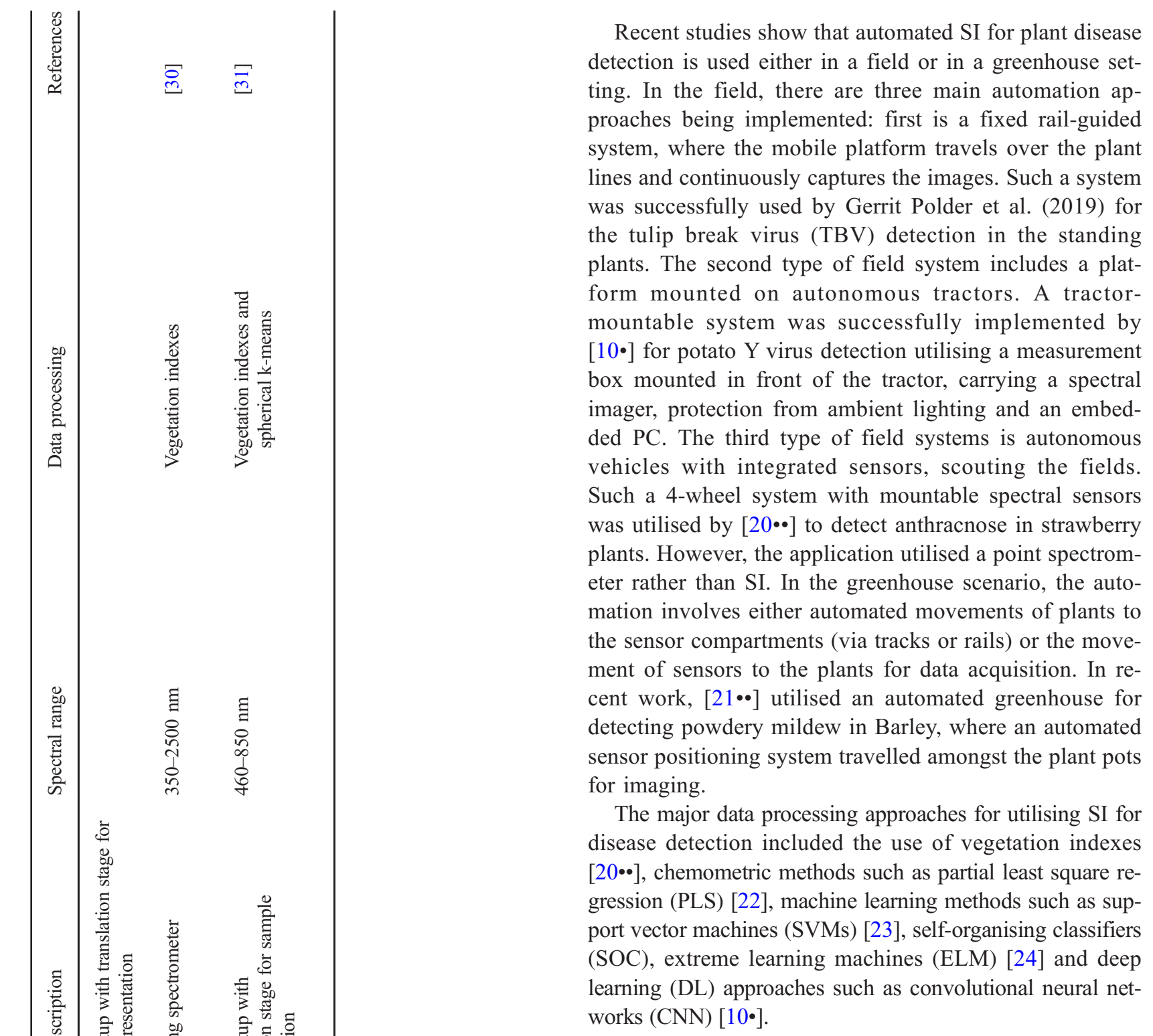

\section{Discussion and Outlook}

A range of applications related to the use of SI in the autonomous platform can be found; however, it can be understood from Table 1 that for the imaging applications, the spectral range used was mainly in the VNIR range $(\sim 400-1000 \mathrm{~nm})$. There are two reasons for this. First, spectral cameras in the complete spectral range $(400$ $2500 \mathrm{~nm})$ are not available in the market. Sensing in the VNIR (400-1000 nm) and the SWIR (1000-2500 nm) range requires different electronic detectors, meaning multiple cameras need to be purchased and applied. Second, the cost of SWIR is almost 5 times compared with the VNIR sensors, making the VNIR the preferred choice for integration with the autonomous setups. Furthermore, VNIR range has the benefit that it captures 
the signal of photosynthetic pigments, which is present only in the spectral range of about $400-700 \mathrm{~nm}$, as well as the 3 rd overtones of proteins, fats and moisture present in the range of 700-1000 $\mathrm{nm}$.

Due to the possibility of SI to capture a wide range of the physicochemical parameter, SI can be used for untargeted plants disease detection, thus not looking for specific pathogens but rather capturing the overall symptoms or changes. This has a major benefit over destructive chemical methods which are focussed on identifying specific pathogens responsible for the damage. The main benefit of SI is its ability to non-destructively capture the spatially distributed spectral properties of plants which can be used to localise the spots of diseases in initial stages which might be missed by destructive analysis. The other major benefit of SI is the ability to perform a real-time detection during acquisition. Real-time detection can be performed by pre-training a model on a small calibration set and then deploying the model onboard an autonomous platform.

A major difficulty with the implementation of SI is the illumination effects caused by the interaction of light with the complex geometry of plants and their surroundings $[12 \bullet \cdot]$. Such an interaction of light causes scattering effects which may mask the real spectral responses. These effects should be corrected for before further data processing. Another challenge is the integration of white reference with the autonomous setup. White reference is required to calculate plant reflectance. Proper positioning of white reference with respect to the plants can reduce major differences in the illumination. However, there is no single solution and the positioning needs to be adapted for different types of plants and their growth stages.

Until now, most of the SI utilised line-scan cameras; however, new snapshot SI systems are emerging in the market which opens possibilities for easy implementation of SI to autonomous setups. In a technical perspective, the future direction should be towards an integration of multiple sensors with autonomous setups, such as the two spectral cameras (VNIR and SWIR) and the 3D-shaped sensors. Another step is their information fusion to deal with the illumination effects. Another major trend is the application of advance concepts from the deep learning domain, such as reinforcement learning and recurrent neural networks (RNNs). As one of the major benefits of integrating SI with autonomous platforms, we foresee the early identification of diseases via precision agriculture, which could lead to early application of plant protection products for sustainable farming practices. As presented in the work, currently, there is little literature on disease detection using spectral imaging on autonomous platforms. Given the fast developments in both affordable SI sensors and autonomous platforms, a huge increase in application can be foreseen.

\section{Compliance with Ethical Standards}

Conflict of Interest The authors declare that they have no conflict of interest.

Human and Animal Rights and Informed Consent This article does not contain any studies with human or animal subjects performed by any of the authors.

Open Access This article is licensed under a Creative Commons Attribution 4.0 International License, which permits use, sharing, adaptation, distribution and reproduction in any medium or format, as long as you give appropriate credit to the original author(s) and the source, provide a link to the Creative Commons licence, and indicate if changes were made. The images or other third party material in this article are included in the article's Creative Commons licence, unless indicated otherwise in a credit line to the material. If material is not included in the article's Creative Commons licence and your intended use is not permitted by statutory regulation or exceeds the permitted use, you will need to obtain permission directly from the copyright holder. To view a copy of this licence, visit http://creativecommons.org/licenses/by/4.0/.

\section{References}

Papers of particular interest, published recently, have been highlighted as:

- Of importance

- Of major importance

1. Savary S, Willocquet L, Pethybridge SJ, Esker P, McRoberts N, Nelson A. The global burden of pathogens and pests on major food crops. Nat Ecol Evol. 2019;3:430.

2. Lucas GB, Campbell L. Introduction to plant diseases: identification and management. Berlin: Springer Science \& Business Media; 2012.

3. Fahrentrapp J, Ria F, Geilhausen M, Panassiti B. Detection of gray mold leaf infections prior to visual symptom appearance using a five-band multispectral sensor. Front Plant Sci. 2019;10. https://doi. org/10.3389/fpls.2019.00628.

4. Sankaran S, Mishra A, Ehsani R, Davis C. A review of advanced techniques for detecting plant diseases. Comput Electron Agric. 2010;72:1-13.

5. Martinelli F, Scalenghe R, Davino S, Panno S, Scuderi G, Ruisi P, et al. Advanced methods of plant disease detection. A review. Agron Sustain Dev. 2015;35:1-25.

6. Ali MM, Bachik NA, Muhadi NA, Yusof TNT, Gomes C. Nondestructive techniques of detecting plant diseases: a review. Physiol Mol Plant Pathol. 2019;108. https://doi.org/10.1016/j.pmpp.2019. 101426.

7. Virlet N, Sabermanesh K, Sadeghi-Tehran P, Hawkesford MJ. Field scanalyzer: an automated robotic field phenotyping platform for detailed crop monitoring. Funct Plant Biol. 2017;44:143-53.

8. Shakoor N, Lee S, Mockler TC. High throughput phenotyping to accelerate crop breeding and monitoring of diseases in the field. Curr Opin Plant Biol. 2017;38:184-92.

9. Polder G, Westeringh Nvd, Kool J, Khan HA, Kootstra G, Nieuwenhuizen A. Nieuwenhuizen, Automatic detection of tulip breaking virus (TBV) using a deep convolutional neural network. IFAC-PapersOnLine. 2019;52:12-7. Use of novel deep learning in spectral imaging for detecting plant disease. 
10. Polder G, Blok PM, de Villiers HAC, van der Wolf JM, Kamp J. Potato virus $\mathrm{Y}$ detection in seed potatoes using deep learning on hyperspectral images. Front Plant Sci. 2019;10. https://doi.org/10. $3389 /$ fpls.2019.00209. Use of novel deep learning in spectral imaging for detecting plant disease.

11. Li L, Zhang Q, Huang DF. A review of imaging techniques for plant phenotyping. Sensors-Basel. 2014;14:20078-111.

12.• Mishra P, Asaari MSM, Herrero-Langreo A, Lohumi S, Diezma B, Scheunders P. Close range hyperspectral imaging of plants: a review. Biosyst Eng. 2017;164:49-67. Key review paper describing the general application of spectral imaging in plant analysis.

13. Lowe A, Harrison N, French AP. Hyperspectral image analysis techniques for the detection and classification of the early onset of plant disease and stress. Plant Methods. 2017;13. https://doi.org/10. 1186/s13007-017-0233-Z.

14. Singla J, Krattinger SG. Biotic stress resistance genes in wheat, in: reference module in food science. Amsterdam: Elsevier; 2016.

15. Madani B, Mirshekari A, Imahori Y. Chapter 19 - physiological responses to stress. In: Yahia EM, editor. Postharvest Physiology and Biochemistry of Fruits and Vegetables. Sawton: Woodhead Publishing; 2019. p. 405-23.

16. Jones JD, Dangl JL. The plant immune system. Nature. 2006;444: 323-9.

17. Jacquemoud S, Verhoef W, Baret F, Bacour C, Zarco-Tejada PJ, Asner GP, et al. PROSPECT plus SAIL models: a review of use for vegetation characterization. Remote Sens Environ. 2009;113: S56-66.

18. Wahabzada M, Mahlein AK, Bauckhage C, Steiner U, Oerke EC, Kersting K. Metro maps of plant disease dynamics-automated mining of differences using hyperspectral images. PLoS One. 2015;10: $1-20$.

19. Mahlein AK. Plant disease detection by imaging sensors-parallels and specific demands for precision agriculture and plant phenotyping. Plant Dis. 2016;100:241-51.

20.• Lu J, Ehsani R, Shi Y, Abdulridha J, de Castro AI, Xu Y. Field detection of anthracnose crown rot in strawberry using spectroscopy technology. Comput Electron Agric. 2017;135:289-99. The study utilizes autonomous platform.

21.• Thomas S, Behmann J, Steier A, Kraska T, Muller O, Rascher U, et al. Quantitative assessment of disease severity and rating of barley cultivars based on hyperspectral imaging in a non-invasive, automated phenotyping platform. Plant Methods. 2018;14:1-12. The study utilizes autonomous platform.

22. Gorretta N, Nouri M, Herrero A, Gowen A, Roger J. Early detection of the fungal disease "apple scab" using SWIR hyperspectral imaging, In: 2019 10th Workshop on Hyperspectral Imaging and Signal Processing: Evolution in Remote Sensing (WHISPERS), 2019, pp. 1-4.

23. Kong WW, Zhang C, Huang WH, Liu F, He Y. Application of hyperspectral imaging to detect Sclerotinia sclerotiorum on oilseed rape stems. Sensors (Basel). 2018;18(1). https://doi.org/10.3390/ s18010123.

24. Xie C, Shao Y, Li X, He Y. Detection of early blight and late blight diseases on tomato leaves using hyperspectral imaging. Sci Rep. 2015;5:16564.

25. Kuska MT, Behmann J, Namini M, Oerke EC, Steiner U, Mahlein AK. Discovering coherency of specific gene expression and optical reflectance properties of barley genotypes differing for resistance reactions against powdery mildew. PLoS One. 2019;14. https://doi. org/10.1371/journal.pone.0213291.

26. Yeh YH, Chung WC, Liao JY, Chung CL, Kuo YF, Lin TT. Strawberry foliar anthracnose assessment by hyperspectral imaging. Comput Electron Agric. 2016;122:1-9.

27. Oerke E-C, Herzog K, Toepfer R. Hyperspectral phenotyping of the reaction of grapevine genotypes to Plasmopara viticola. J Exp Bot. 2016;67:5529-43.

28. Jarolmasjed S, Sankaran S, Marzougui A, Kostick S, Si Y, Quirós Vargas JJ, et al. High-throughput phenotyping of fire blight disease symptoms using sensing techniques in apple. Front Plant Sci. 2019;10. https://doi.org/10.3389/fpls.2019.00576.

29. Lee H, Kim MS, Lim H-S, Park E, Lee W-H, Cho B-K. Detection of cucumber green mottle mosaic virus-infected watermelon seeds using a near-infrared (NIR) hyperspectral imaging system: application to seeds of the "Sambok honey" cultivar. Biosyst Eng. 2016;148:138-47.

30. Lu JZ, Ehsani R, Shi YY, de Castro AI, Wang S. Detection of multitomato leaf diseases (late blight, target and bacterial spots) in different stages by using a spectral-based sensor. Sci Rep. 2018;8. https://doi.org/10.1038/s41598-018-21191-6.

31. Leucker M, Wahabzada M, Kersting K, Peter M, Beyer W, Steiner $\mathrm{U}$, et al. Hyperspectral imaging reveals the effect of sugar beet quantitative trait loci on Cercospora leaf spot resistance. Funct Plant Biol. 2017;44:1-9.

Publisher's note Springer Nature remains neutral with regard to jurisdictional claims in published maps and institutional affiliations. 